\title{
CARCINOMA BASALÓIDE ESCAMOSO: UMA FORMA RARA E AGRESSIVA DE CÂNCER DO ESÔFAGO E REVISÃO DA LITERATURA
}

\section{Basaloid squamous carcinoma of the esophagus: a rare and aggressive form of esophageal cancer and literature review}

\author{
Maria Aparecida Coelho de Arruda HENRY, Mauro Masson LERCO, \\ Walmar Kerche de OLIVEIRA, Maria Aparecida Marchesan RODRIGUES
}

ABCDDV/527

Henry MACA, Lerco MM, Oliveira WK, Rodrigues MAM. Carcinoma basalóide escamoso: uma forma rara e agressiva de câncer do esôfago e revisão da literatura. ABCD Arq Bras Cir Dig 2007;20(1):62-4.

RESUMO - Introdução - O carcinoma basalóide escamoso ocorre com maior freqüência no trato aerodigestivo superior e raramente acomete o esôfago. Relato do caso - Dos 134 pacientes com câncer do esôfago atendidos no Hospital Universitário de Botucatu-Unesp, São Paulo, Brasil,de 1990 a 1999, somente um paciente $(0,74 \%)$ apresentou carcinoma basalóide escamoso do esôfago. Tratava-se de paciente masculino, 41 anos, branco, lavrador com disfagia, regurgitação e emagrecimento há três meses. Referia tabagismo e etilismo há muitos anos. O esofagograma e o exame endoscópico revelaram lesão vegetante no terço distal do esôfago. A biópsia demonstrou neoplasia intraepitelial de alto grau associada a blocos de células basalóides que infiltravam o cório da mucosa, caracterizando o carcinoma basalóide escamoso. Os marcadores imunoistoquímicos foram positivos para o antígeno carcinoembriônico e para citoceratinas de alto peso molecular. A tomografia computadorizada revelou múltiplas metástases nos pulmões, fígado, e nódulos linfáticos regionais, documentando a fase avançada de evolução da doença. O tratamento consistiu apenas na realização de gastrostomia. O paciente apresentou queda acentuada do estado geral e evoluiu para óbito com quadro de melena quatro meses após o diagnóstico. Conclusão - O carcinoma basalóide escamoso é uma forma rara e agressiva de câncer do esôfago e o prognóstico depende do estadiamento da lesão e das condições clínicas do paciente no momento do diagnóstico.

DESCRITORES - Neoplasias esofágicas. Carcinoma.

\section{INTRODUÇÃO}

O carcinoma basalóide escamoso (CBE) é uma variante incomum do carcinoma de células escamosas do esôfago (CEC), com características clínicas próprias, sendo a alta agressividade biológica e o mau prognóstico os atributos de maior destaque ${ }^{2,4,10}$. Este tipo de neoplasia ocorre com maior freqüência no trato aerodigestivo superior, tendo como sítios primários preferenciais a cavidade oral, faringe, laringe, seio piriforme e traquéia ${ }^{5,8,14}$. A incidência desta lesão no esôfago é extremamente rara, corresponde a 0,1 a $5 \%$ das neoplasias malignas do esôfago ${ }^{1,6,11,15}$.

O objetivo desta comunicação é apresentar os aspectos clínico-patológicos de um paciente com CBE primário do esôfago, considerando a raridade deste tipo de câncer e seus atributos biológicos, nesta topografia do trato gastrointestinal.

Trabalho realizado na Faculdade de Medicina de Botucatu - Unesp, Botucatu, SP, Brasil.

Endereço para correspondência: Maria Aparecida Coelho de Arruda Henry, e-mail: rhenry@ibb.unesp.br

\section{RELATO DO CASO}

Masculino, 41 anos, branco, lavrador, natural e procedente de São Manuel (SP). Referia disfagia para sólidos há três meses, regurgitação e emagrecimento. Fumava três cigarros de palha por dia há muitos anos e dos 15 aos 24 anos ingeria um litro de aguardente diariamente. Regular estado geral, mucosas descoradas ++ , desnutrido. O esofagograma demonstrou falha de enchimento nos $12 \mathrm{~cm}$ distais do esôfago (Figura 1). A endoscopia digestiva alta confirmou a presença de lesão vegetante, ulcerada a 31 $\mathrm{cm}$ da arcada dentária superior, obstrutiva. O estudo histopatológico demonstrou carcinoma basalóide escamoso (Figura 2) e a avaliação imunoistoquímica resultou-se positiva para o antígeno carcinoembriônico e citoqueratinas de alto peso molecular. A tomografia computadorizada demonstrou metástases pulmonares, hepáticas e adenomegalia retroperitoneal. Frente ao estadiamento avançado do tumor (Tx N1 M1: Estadio IV), optou-se pela realização de gastrostomia. O paciente apresentou acentuada queda do estado geral e evoluiu para óbito com quadro de melena, quatro meses após o diagnóstico. 


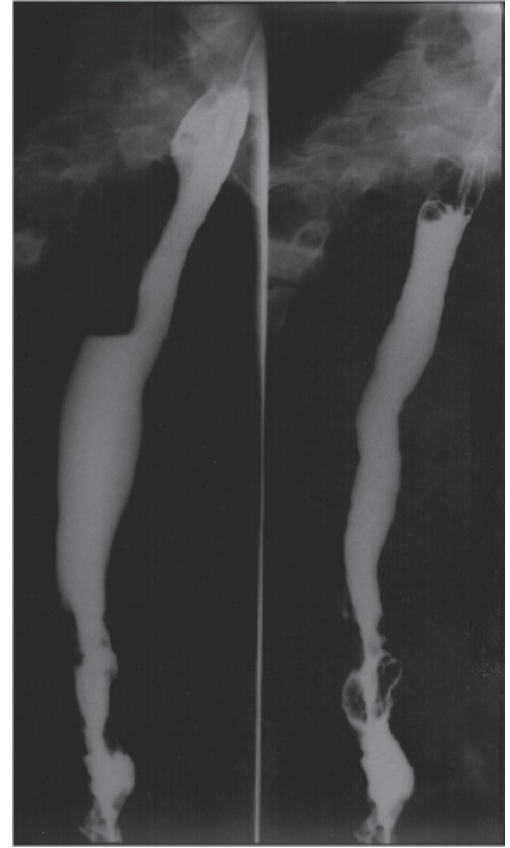

FIGURA 1 - Esofagograma demonstrando irregularidades da mucosa nos $12 \mathrm{~cm}$ distais do esôfago

\section{DISCUSSÃO}

A exemplo do que ocorre com as neoplasias malignas do esôfago $0^{9,12,16}$, o carcinoma basalóide escamoso é mais freqüente em homens com idade acima de 40 anos e no terço médio do esôfago torácico ${ }^{2,4,5,13}$. Tais características sobrepõem-se às observadas no presente caso, exceto para o local da lesão, que estava situada no terço distal do esôfago, coincidindo com o referido por Albuquerque et al (2006) ${ }^{1}$. Identificou-se somente um caso de CBE $(0,74 \%)$ entre 134 casos de câncer do esôfago diagnosticados e tratados no Hospital Universitário de Botucatu - Unesp, São Paulo, na década de 90. Esta forma rara e agressiva de câncer do esôfago tem sido relatada na literatura com incidência de 0,1 a $5 \%$ dos carcinomas do esôfago.

O CBE foi descrito por Wain et $\mathrm{al}^{14}$. em 1986, que estabeleceram os critérios histopatológicos para o diagnóstico desta forma de câncer do esôfago. Os atributos morfológicos incluem a presença de células com núcleos basalóides, dispostos em paliçada na periferia de blocos tumorais, alto índice de mitoses e áreas de necrose. A análise histopatológica do presente caso demonstrou todos estes atributos morfológicos, caracterizando o CBE. O estudo imunoistoquímico revelou que as células neoplásicas apresentavam imunoreatividade para citoceratinas de alto peso molecular e para o antígeno carcinoembriônico, confirmando a histogênese epitelial da lesão.

Vários fatores têm sido implicados na patogênese deste tumor, sendo os mais citados o alcoolismo, tabagismo, irradiação, vírus de Epstein Barr e o vírus do papiloma. A teoria mais aceita é a de Ho et al. ${ }^{3}$ (1984), que propõe que o CBE origina-se de células primitivas da camada basal

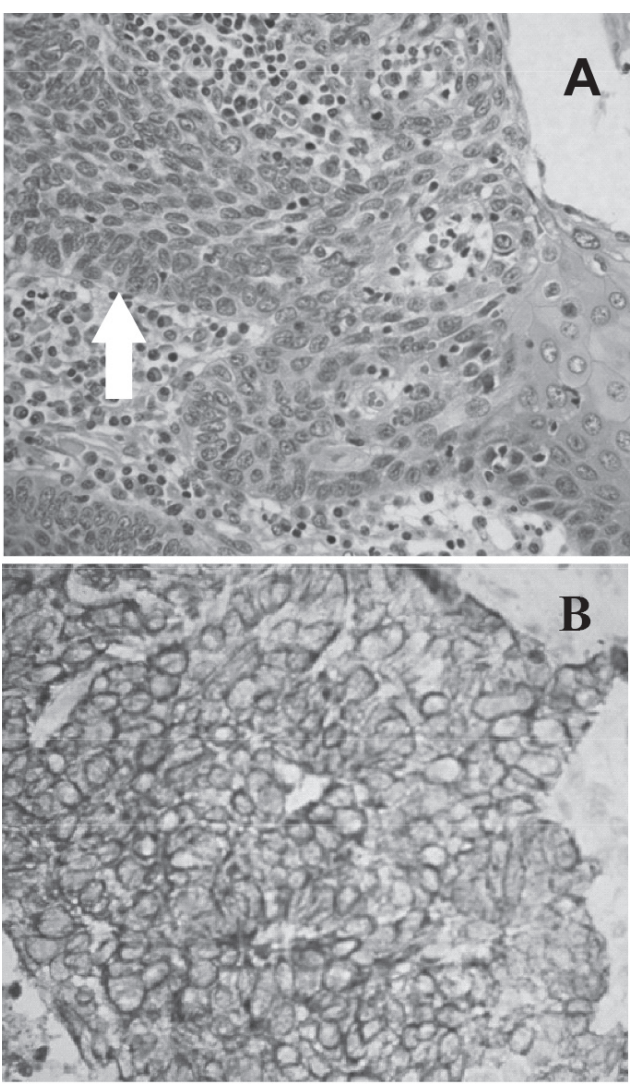

FIGURA 2 - Características histopatológicas (A) e imunoistoquímicas (B) do carcinoma basalóide-escamoso

do epitélio pavimentoso que reveste a mucosa do esôfago. Estas células totipotentes podem apresentar diferentes padrões de diferenciação, para a linhagem escamosa (CEC), glandular (adenocarcinoma), neuroendócrina (carcinoma de pequenas células) e basalóide (CBE).

Quanto à agressividade biológica, o CBE ocupa posição intermediária entre o carcinoma de células escamosas (CEC) e o carcinoma de pequenas células, sendo mais agressivo que o CEC e menos agressivo do que o carcinoma de pequenas células ${ }^{15}$. Contrapõe-se a esta proposição o estudo de Sarbia et al. ${ }^{13}$ (1997) que analisaram comparativamente a evolução de 17 pacientes com CBE e 133 pacientes com CEC. Os autores observaram que a agressividade biológica foi similar para ambos os tipos de tumores.

Noguchi et al. ${ }^{10}(2003)$ relataram que as metástases hematogênicas para o fígado e pulmões são comuns neste tipo de tumor, fato que ocorreu com este paciente e inviabilizou a indicação da ressecção cirúrgica do esôfago. Entretanto, é possível diagnosticar este tipo de neoplasia em fase precoce de evolução. Albuquerque et al. ${ }^{1}$ (2006) identificaram por ultrassonografia endoscópica lesão limitada à mucosa, com invasão superficial da submucosa. A conduta adotada foi a ressecção endoscópica da lesão, com sucesso.

O tratamento do CBE segue os princípios oncológicos gerais adotados para as neoplasias malignas do trato gastrointestinal. Autores japoneses preconizam quimioterapia com 5-Fluoracil e cisplatina para os tumores localmente 
avançados, sem metástases à distância ${ }^{4,7}$. Esta conduta visa reduzir as dimensões da lesão primária, de modo a viabilizar a realização da esofagectomia associada a linfadenectomia em três campos: cervical, torácico e abdominal, com razoável índice de sobrevida. No presente caso tendo em vista o estadio avançado de evolução da doença, com metástases disseminadas (Estadio IV) e o grave comprometimento do estado nutricional do paciente, optou-se apenas pela realização de uma via alternativa para suporte nutricional (gastrostomia), realizada com anestesia local.

\section{CONCLUSÃO}

O carcinoma basalóide escamoso é uma forma rara e agressiva de câncer do esôfago, distinta do carcinoma de células escamosas, sendo seu prognóstico relacionado à extensão da lesão no hospedeiro e às condições clínicas do paciente no momento do diagnóstico.

Henry MACA, Lerco MM, Oliveira WK, Rodrigues MAM. Basaloid squamous carcinoma of the esophagus: a rare and aggressive form of esophageal cancer and literature review. ABCD Arq Bras Cir Dig 2007; 20(1):62-4.

ABSTRACT - Background - Basaloid squamous carcinoma is more frequently found in the upper aerodigestive tract, being rarely found in the esophagus. Case report - Of a total of 134 esophagus cancer patients in the Hospital Universitário de Botucatu-Unesp, in São Paulo, from 1990 through 1999 , only one patient $(0,74 \%)$, presented the basaloid squamous carcinoma of the esophagus. This patient, a 41 year-old caucasian male farmer, presented dysfagia, regurgitation and weight loss for the last three months, being a smoker and alcoholic for many years. Endoscopy and esophagram revealed a vegetative lesion in the distal region of the esophagus. Biopsy showed a high-grade intra-epithelial neoplasm associated with basaloid cells infiltrating the corian mucosa, characteristic of the squamous basaloid carcinoma. Immunohistochemical markers were positive for carcinoembrionary antigen and high molecular weight citokeratins. Computerized tomography revealed multiple metastasis in the lungs, liver and regional lymphatic nodules, all evidence of an advanced evolution of the disease. Treatment consisted of gastrostomy. The patient presented an accentuated fall pertaining it's general state and died with a state of melena four months after diagnosis. Conclusion - The basaloid squamous carcinoma is a rare and aggressive form of esophagus cancer and the prognosis depends in the state at which the lesion is and in the clinical conditions of the patient at the time of diagnosis.

HEADINGS - Esophageal neoplasms. Carcinoma.

\section{REFERÊNCIAS}

1. Albuquerque W, Arantes V, Roda R, Maciel DP, Resende CC, Rodrigues MAG, Nogueira AM. Ressecção endoscópica de carcinoma basalóide escamoso do esôfago. GED 2006; 25 (Supl 1): 173- 4.

2. Cho KJ, Jang JJ, Lee SS, Zo JI. Basaloid squamous carcinoma of the oesophagus: a distint neoplasm with multipotential differentiation. Histopathology 2000; 36: 331-40

3. Ho KJ, Herrera GA, Jones MJ. Small cell carcinoma of the esophagus: evidence for a unified histogenesis . Hum Pathol 1984; 15-460-4.

4. Hyodo M, Hosoya Y, Arai W, Yokoyama T, Shibusawa H, Yasuda Y, Nagai H, Shibayama C, Nakazawa M. Preoperative chemoradiotherapy and surgery for locally advanced esophageal basaloid squamous carcinoma: report of successful. Esophagus 2005; 2:85-9.

5. Kato T, Morita T, Fujita M, Miyasaka Y, Horita S, Watanabe Y, Kato H. Basaloid squamous carcinoma of the esophagus: report of a case. Surg Today 2000; 30: 163-7.

6. Kawamoto M, Kunisaki C, Kunihiro O, Kamiya N, Moriwaki Y, Akiyama H, Shimada H, Kono N, Nakatami Y, Kunisaki R. Basoloid cell carcinoma of the esophagus with a metastatic neck tumor of unknown origin: report of a case. Surg Today 2003; 33: 529-32.

7. Koide N, Kishimoto K, Nakamura T, Igarashi J, Maezawa T, Kanazaki K, Adachi W, Amano J. Basaloid squamous carcinoma of the esophagus treated by preoperative chemotherapy: report of two cases. Surg 2003; 33: 444-7.

8. Luna MA, Naggar AE, Parichatikanond P, Weber RS, Batsakis JG. Basaloid squamous carcinoma of the upper aerodigestive tract. Cancer 1990; 66: 537 42.
9. Mustard RA \& Ibberson O. Carcinoma of the esophagus. A review of 381 cases admitted to Toronto General Hospital 1937-1953 inclusive. Ann Surg 1956; 144:927-40.

10. Noguchi H, Naomoto Y, Haisa M, Yamatsuji T, Shigemitsu K, Shirakawa Y, Kataoka M, Ohkawa T, Nobuhisa T, Kobayashi M, Gunduz M, Tanaka N. Two cases of superficial basaloid squamous carcinoma of the esophagus. Dis Esophagus 2003; 16: 342-5.

11. Preto J, Pimenta A, Gouveia A, Magalhães A, Valbuena C, Cruz D, Sousa J, Ferreira S, Ferreira A, Alves O, Rodrigues S, Oliveira MC. Basaloid-squamous carcinoma of the esophagus - a study of 4 cases. Anais 10 World Congress of International Society for Diseases of the Esophagus. Adelaide-Austrália 2006, p.50.

12. Rosa AR, Gurski RR, Schirmer CC, Brentano L, Kruel CD. Survival and prognostic factors in patients with resected epidermoid oesophageal carcinoma. Int Surg 1999; 84:193-8.

13. Sarbia M, Verreet P, Bettinger F, Dutkowski P, Heep H, Willers R, Gabbert HE. Basaloid squamous cell carcinoma of the esophagus. Diagnosis and prognosis. Cancer 1997;79:1871-78.

14. Wain SL, Kier R, Vollmer RT, Bossen EH. Basaloid squamous-carcinoma of the tongue, hypopharynx, and larynx: report of 10 cases. Hum Pathol 1986;17: 1158-66.

15. Zhang XH, Sun GH, Zhou XJ, Guo HF, Zhang TH. Basaloid squamous carcinoma of esophagus: a clinicopathological, immunohistochemical and electron microscopic study of sixteen cases. World J Surg 1998; 4: 397-403.

16. Zilberstein B, Faintuch J, Pinotti HW. Câncer do esôfago. In:Pinotti HW. Tratado de clínica cirúrgica do aparelho digestivo. São Paulo:Atheneu:1994. Cap 15, p. 416-25.

Conflito de interesse: não há

Fonte financiadora: não há

Recebido para publicação em: 10/09/2006 Aceito para publicação em: 05/12/2006 